\title{
Fragile X Mental Retardation Protein Shifts between Polyribosomes and Stress Granules after Neuronal Injury by Arsenite Stress or In Vivo Hippocampal Electrode Insertion
}

\author{
Soong Ho Kim, ${ }^{1,2}$ Willie K. Dong, ${ }^{1}$ Ivan Jeanne Weiler, ${ }^{1}$ and William T. Greenough ${ }^{1,2,3}$ \\ ${ }^{1}$ Beckman Institute, ${ }^{2}$ Neuroscience Program, and ${ }^{3}$ Departments of Psychology, Psychiatry, and Cell and Developmental Biology, University of Illinois at \\ Urbana-Champaign, Urbana, Illinois 61801
}

\begin{abstract}
Fragile X mental retardation protein (FMRP), the lack of which causes fragile X syndrome, is an RNA-binding protein encoded by the FMR1 gene. FMRP accompanies mRNAs from the nucleus to dendritic regions and is thought to regulate their translation at synapses. It has been shown that FMRP moves into nontranslating stress granules (SGs) during heat stress of cultured fibroblasts (Mazroui et al., 2002). We used a novel method to isolate SGs from neurons by virtue of their TIA-1 (T-cell intracellular antigen 1) protein component, and found that FMRP moved out of polyribosomes and into SGs subsequent to oxidative stress. We then examined FMRP changes in subcellular localization resulting from mechanically induced neuronal injury by placement of electrodes into the dentate gyrus and the perforant path of the hippocampus in vivo. During the first $10 \mathrm{~min}$ after electrode insertion into one hippocampus, FMRP shifted into SGs and away from polyribosomes, in both hippocampi. Although the injury discharge subsided beyond $10 \mathrm{~s}$, FMRP levels in polyribosomes and stress granules did not return to basal levels until $30 \mathrm{~min}$ after electrode penetration. Our findings suggest that procedures for in vivo induction of long-term potentiation or long-term depression should incorporate a $30 \mathrm{~min}$ rest period after electrode insertion, and indicate that the contralateral hippocampus cannot be considered an unstimulated control tissue.
\end{abstract}

Key words: FMRP; stress granules; brain injury; protein translation; LTP; ischemia

\section{Introduction}

Fragile X mental retardation syndrome (FXS) is an inherited, X-linked disorder, affecting 1:4000 males (Turner et al., 1996). In most cases, expansion of the CGG trinucleotide repeat in the $5^{\prime}$-untranslated region of the gene and subsequent methylation of its CpG island causes transcriptional silencing, leading to the functional absence of fragile $\mathrm{X}$ mental retardation protein (FMRP) (Warren and Nelson, 1994). Brains of FXS patients as well as mouse knock-out models exhibit abnormal dendritic spine shapes, numbers, and pruning deficits, suggesting that the protein plays an important role in activity-dependent synaptic maturation and plasticity (Irwin et al., 2002). Mouse models of FXS showed reduced cerebral cortical long-term potentiation (LTP) (Li et al., 2002) and enhanced hippocampal long-term

\footnotetext{
Received Aug. 31, 2005; revised Jan. 12, 2006; accepted Jan. 14, 2006.

This work was supported by the FRAXA Research Foundation, the Illinois-Eastern lowa District of Kiwanis International Spastic Paralysis Research Foundation, and National Institutes of Health Grants MH35321, AG10154, and MH64272-01. We thank Dr. Scott J. Robinson (Imaging Technology Group, Beckman Institute, Urbana, IL) for assistance in TEM and Dr. James D. Churchill (St. Louis University, St. Louis, MO) for his contribution to preliminary work. We are grateful to Dr. Jean-Louis Mandel (Institut de Génétique et de Biologie Moléculaire et Cellulaire, Strasbourg, France) for 1 C 3 antibody; Dr. André Hoogeveen (Erasmus University, Rotterdam, The Netherlands) for antibodies to FXR1 and FXR2; Dr. Peter Vanderklish (The Scripps Research Institute, La Jolla, CA) for antibodies to L3, L4, and L7a; Dr. Stephanie Ceman (University of Illinois, Urbana, IL) for anti-FMRP antibody 7G1-1; and Dr. Barbara Bardoni (Université de Nice, Nice, France) for antibody to NUFIP.

Correspondence should be addressed to Soong Ho Kim, Beckman Institute, 405 North Mathews Avenue, Urbana, IL 61801. E-mail: kanai@uiuc.edu.

DOI:10.1523/JNEUROSCI.3680-05.2006

Copyright $\odot 2006$ Society for Neuroscience $\quad 0270-6474 / 06 / 262413-06 \$ 15.00 / 0$
}

depression (LTD), but normal hippocampal LTP (Huber et al., 2002). FMRP is an RNA-binding protein; it binds to a subset of mRNAs (Miyashiro et al., 2003).

It is known that cells exposed to stress such as UV light, starvation, double-stranded RNA, or hyperosmolarity shut down translation of housekeeping mRNAs while upregulating translation of stress-induced transcripts such as heat shock protein mRNAs (Nover et al., 1989). The housekeeping mRNAs are sequestered in cytoplasmic microdomains called stress granules (SGs), which contain two specific RNA-binding proteins, T-cell intracellular antigen 1 (TIA-1) and TIA-1-related protein (TIAR), not present in polyribosomes (Kedersha et al., 1999). Interestingly, it has been shown by Mazroui et al. (2002) that, in cultured fibroblasts, FMRP moves from polyribosomes into stress granules during application of stress such as heat. In patients, translational arrest after brain ischemia has recently been shown to be mediated by stress granules (Kayali et al., 2005).

We used a TIA-1 antibody to immunopurify stress granules from gently lysed hippocampal neurons using magnetic beads. This made it possible to obtain a highly enriched population of stress granules without including other granules of similar physical characteristics that have a different function. We showed that these granules share the size and protein composition characteristic of classical stress granules. We measured the amounts of FMRP associated with stress granules and with polyribosomes, under various conditions. After verifying that FMRP shifts into stress granules in response to oxidative stress, using arsenite to 
treat hippocampal tissue, we next examined the behavior of FMRP in hippocampal cells subsequent to in vivo electrode insertion.

We used simple electrode insertion into the dentate gyrus (DG) and perforant path (PP) of rat hippocampus to mechanically induce injury in neuronal tissue. A systematic analysis of TIA-1-bearing stress granules, compared with polyribosomes, suggests that there was rapid, active migration of FMRP out of polyribosomes and into TIA-1 granules in reaction to the stress accompanying electrode insertion. The injury discharge, which subsided beyond $10 \mathrm{~s}$, was strong enough to trigger an immediate shift of FMRP from polyribosomes to stress granules, peaking at $10 \mathrm{~min}$, followed by return to baseline values at $30 \mathrm{~min}$. These alterations were observed in both hippocampi, although electrodes were inserted only on one side; thus, the injury discharge most likely crosses the hippocampal commissures, resulting in parallel cellular stress responses in both cell populations.

\section{Materials and Methods}

Animals. Twelve adult male rats (Long-Evans), $>250 \mathrm{~g}$ and 65 postnatal days of age, were used for insertion of electrodes into the right hippocampus (in vivo) and subsequent removal of both hippocampi for biochemical analysis. Four groups of rats (three in each) were used for electrode insertion and placement at target sites for varying lengths of time $(10,20$, 30 , and $60 \mathrm{~min}$ ). An additional seven rats were used as control animals; four were decapitated without anesthesia and electrode insertion $(\mathrm{C} 1)$, and three were anesthetized but no electrodes were inserted into the hippocampus (C2). Twenty wild-type mice of the FVB.129P2-fmr1 ${ }^{\mathrm{tm} 1 \mathrm{Cgr}}$ strain, 10-14 d of age, were used for treatment of the hippocampus with arsenite (in vitro) and subsequent biochemical analysis, and to prepare granules for analysis by transmission electron microscopy (TEM). Six young rats (Long-Evans), 10-14 d of age, were used to study the proportional distribution of FMRP in granules.

Stress induction by arsenite. To provide a model of classical chemical stress to compare with hippocampi subjected to mechanical stress or injury, we placed rat hippocampi into aerated DMEM at $37^{\circ} \mathrm{C}$ for $10 \mathrm{~min}$ with or without $200 \mu \mathrm{M}$ arsenite. Hippocampi were then frozen quickly on dry ice and processed in the same manner as hippocampi from the electrode insertion experiments.

Preparation of cellular subfractions and Western blots. Immediately after chemical stress or electrode insertion and recording, hippocampi were frozen on dry ice. NE-PER nuclear and cytoplasmic extraction reagents (Pierce Biotechnology, Rockford, IL) were used to manually homogenize hippocampi and separate nuclear and cytoplasmic fractions. The cytoplasmic fraction was centrifuged at $12,000 \times g$ for 10 min to collect granules. Supernatants were layered over $1 \mathrm{M}$ sucrose in Ross-Kobs polysome buffer ( $1 \mathrm{~mm}$ potassium acetate, $10 \mathrm{~mm}$ Tris- $\mathrm{HCl}, \mathrm{pH} 7.6,1.5 \mathrm{~mm}$ $\mathrm{MgCl}_{2}$ ) and then centrifuged for $11 \mathrm{~min}$ at $400,000 \times g$ at $4^{\circ} \mathrm{C}$. The resulting polyribosomal pellet was resuspended in $\mathrm{Li}^{+}$lysis buffer $[1 \%$ NP-40, 50 mm Tris-HCl, pH 8.0, $100 \mathrm{~mm} \mathrm{LiCl,} \mathrm{protease} \mathrm{inhibitor} \mathrm{mixture}$ (Sigma, St. Louis, MO), $0.1 \mathrm{~mm}$ sodium orthovanadate, and $0.1 \mu \mathrm{M}$ okadaic acid].

The pelleted granule fraction was resuspended in low-salt lysis buffer (1\% NP-40, 50 mm Tris-HCl, pH 8.0), mixed with Protein G Magnetic Microbeads (Miltenyi Biotec, Auburn, CA), which were preincubated with goat anti-TIA-1 antibody (Santa Cruz Biotechnology, Santa Cruz, $\mathrm{CA}$ ) and BSA, and incubated on ice for $1 \mathrm{~h}$. Immunoprecipitates were captured using $\mu \mathrm{MACS}$ columns (Miltenyi Biotec). After washing four times in low-salt lysis buffer and once in low-salt washing buffer (200 mM Tris- $\mathrm{HCl}, \mathrm{pH} 7.5)$, proteins were released by hot SDS elution buffer $(0.4 \%$ SDS, 13 mm Tris- $\mathrm{HCl}, \mathrm{pH} 6.8,3 \%$ glycerol, $1 \%$ $\beta$-mercaptoethanol, $0.005 \%$ bromphenol blue). As a control, lysates were treated with nonimmune goat IgG and magnetic beads. To exclude a possibility of unspecific binding of protein to $\mu \mathrm{MACS}$ columns, lysates were run through the columns without an antibody or magnetic beads, followed by washing and elution in the same manner.

As a control for nonspecific coaggregation of FMRP with TIA-1, ly- sates were immunoprecipitated with 7G1-1, a monoclonal antibody to FMRP (a gift from Dr. Stephanie Ceman, University of Illinois, Urbana, IL), and as an isotype control, with mouse IgG2a; the presence of TIA-1 was tested in both samples.

Protein samples were separated on an $8 \%$ acrylamide gel, blotted, and incubated overnight at $4^{\circ} \mathrm{C}$ with primary antibody. FMRP was tested with antibody 1C3 (a gift from J.-L. Mandel, Institut de Génétique et de Biologie Moléculaire et Cellulaire, Strasbourg, France). Antibodies to TIA-1, TIAR, Hu antigen R (HuR), eukaryotic translation initiation factor $4 \mathrm{G}$ (eIF4G), and L28 were obtained from Santa Cruz Biotechnology. Antibodies to S6 and eIF4E were from Cell Signaling Technology (Beverly, MA). Antibodies to L3, L4, and L7a were a gift from Dr. Peter Vanderklish (The Scripps Research Institute, La Jolla, CA). Antibody to nuclear FMRP-interacting protein (NUFIP) was a gift from Dr. Barbara Bardoni (Université de Nice, Nice, France). FXR1 and FXR2 were detected with antibody 2107 and 1937, respectively (courtesy of Dr. André Hoogeveen, Erasmus University, Rotterdam, The Netherlands). Horseradish peroxidase-coupled secondary antibodies were detected by chemiluminescence (Pierce Biotechnology or Sigma). As a loading control, each blot was subsequently stained for $\beta$-actin (Sigma) in the same manner.

To quantify protein levels, films were scanned, and relative optical densities were determined using Scion Image Beta 4.02 (Scion, Frederick, MD). Specifically, a square enclosing all four FMRP bands $(78-80 \mathrm{kDa})$ was placed on each lane, and the "mean gray value" for pixels within each square was obtained. For each lane, the background-subtracted value for each band or set of bands was then divided by the value of the background-corrected actin band from the same lane to obtain a normalized value. Each protein sample was run in triplicate on three separate blots, and normalized values from each set of replications were averaged to obtain a final value for each sample.

Proportional distribution of FMRP in granules. After obtaining a granule pellet by centrifugation of cytoplasm at $12,000 \times g$ for $10 \mathrm{~min}$, the pellet was lysed and aliquoted into two samples; one was saved as the "total granule" sample and the other was used for immunoprecipitation to collect stress granules. FMRP levels in both samples were measured and normalized to total protein level on nitrocellulose membranes stained with amido black.

TEM. Stress granules for EM examination were prepared using the same lysis and immunoprecipitation procedures from mouse hippocampi treated with arsenite. However, they were not separated in the $\mu$ MACS column because of difficulty in retrieving them intact from the column, but instead the granules were washed three times gently by standard magnetic bead separation procedures. This washing method is less rigorous than the column, so we expected a small residue of other kinds of granules as well as immunotargeted stress granules. The sample was fixed with $2.5 \%$ glutaraldehyde in low-salt washing buffer, put onto glow-discharged grids, and contrast-enhanced with uranyl acetate. Pictures were taken with a Philips CM200 TEM.

Anesthesia. All rats used for in vivo studies were anesthetized with halothane. Anesthesia was induced with $4 \%$ halothane in oxygen using a vented chamber that allowed excess halogenated gas to be removed. The trachea was intubated, and anesthesia was maintained with $1.5 \%$ inspired halothane in oxygen, delivered through a coaxial breathing apparatus with an excess halothane scavenger attachment.

Surgery. A craniotomy was performed to expose the right caudal portion of the cerebral hemisphere from bregma to occiput and from midline to 5-6 mm lateral. This was done to allow simultaneous, stereotaxic placement of two electrical recording electrodes in the PP and DG of the right hippocampus.

Electrophysiological recording. Multiple cell activity was recorded in the PP and DG using metal, epoxy-insulated bipolar concentric macroelectrodes arranged in a fixed array. The concentric macrorecording electrode consisted of an exposed uninsulated tip used for the active recording lead and the outer exposed ring ( $0.5 \mathrm{~mm}$ from tip) used for the reference recording lead. An electrode impedance of $50 \mathrm{k} \Omega$ across the active and reference leads was measured before brain insertion and was used for recording in all rats. Recordings of multiple cell discharges were made through an AC preamplifier (Grass Instruments, Quincy, MA) 
A

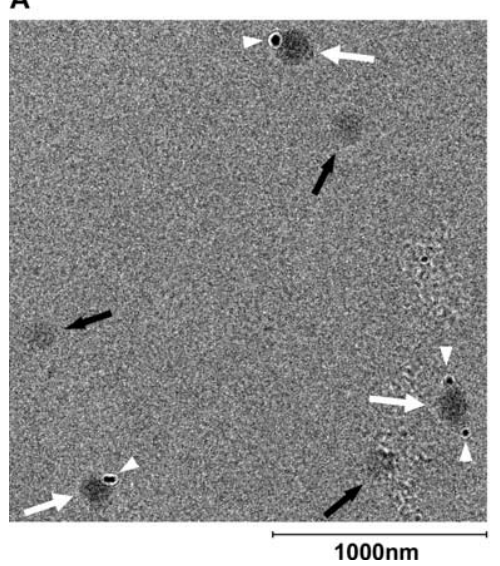

B

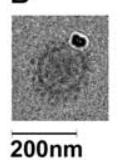

C

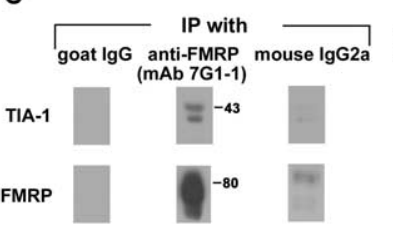

D

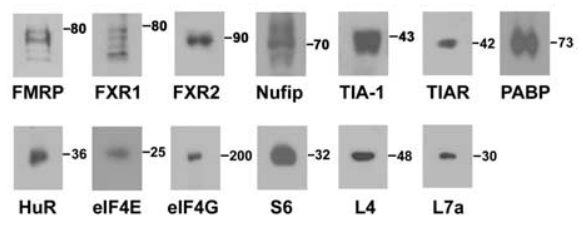

Figure 1. Characterization of SGs prepared with antibody-coupled magnetic microbeads. A, TEM picture of immunoprecipitated stress granules as well as other types of granules. Immunoprecipitation was performed to an essential component of stress granules, TIA-1. Magnetic microbeads (the size is $\sim 50 \mathrm{~nm}$ ) (white arrowheads) selectively bound to TIA-1 granules (SGs) (white arrows) but not to other kinds of granules (transport granules, etc.) (black arrows). Because it is hard to retrieve intact SGs from a $\mu M A C S$ column after the microbeads and the metal column are magnetized, we adopted a conventional magnetic bead separation for TEM procedures using a small PCR tube and washing the sample gently a few times. This washing is less rigorous than using a $\mu$ MACS column; hence a small number of other granules were expected in the preparation for TEM. For quantitative study of FMRP, granules without microbeads (hence, not stress granules) were washed off of the magnetic columns by complete washing. $\boldsymbol{B}$, A magnified SG with a microbead. C, Western blot analysis of immunoprecipitated SGs shows that they have FMRP, FXR1, FXR2, and NUFIP, as well as TIA-1, TIAR, S6, PABP, elF4E, elF4G, HuR, which are known components of SGs. Some large ribosomal protein $(\mathrm{L} 4, \mathrm{L7}$ a) are present in SGs, but not $\mathrm{L} 3$ or L28 (data not shown). D, As a control, immunoprecipitation (IP) with nonimmune goat IgG did not pull down TIA-1 or FMRP. IP for FMRP brought down TIA-1; an isotype control using mouse lgG2a showed almost undetectable amounts of FMRP and TIA-1. A $\mu$ MACS column itself did not attract TIA-1 or FMRP as shown in "no antibody, no beads" control.

with a gain of $1000 \times$ or $2000 \times$ and low- and high-pass filters set at $30 \mathrm{~Hz}$ and $3 \mathrm{kHz}$, respectively. Amplified multicellular discharges (action potentials) were routed to an analog-to-digital signal converter and then displayed and stored on a laptop computer using a Clampex signal processing program (Molecular Devices, Foster City, CA). All recordings were initiated immediately (time " 0 " of recording trace) when the PP and DG electrodes were guided stereotaxically to their target sites.

Discharge analysis. Multiunit discharges recorded from the PP and DG electrodes were analyzed for spike frequency using a Clampfit program (Molecular Devices). We were particularly interested in the discharges of injured neurons at the individual electrode sites (PP or DG) and the possibility that the injury discharges in the PP may influence activity in the DG because the PP axons are connected to DG cells across one synapse in the molecular layer. If the injury discharges in the PP do influence DG activity (in addition to its own DG injury discharges), we wanted to know to what extent. To accomplish that goal we performed a crosscorrelation analysis of the discharges at both sites to determine how well the discharges at one site are time-locked to the discharges at the other site.

Statistical analysis. Significance of changes from control in variables measured was determined using an unpaired $t$ test. SEM was calculated, and a change was considered significant if $p$ was $<0.05$.

\section{Results}

\section{Stress granules}

We used an antibody to the stress granule marker, TIA-1, to isolate defined granules from mouse hippocampi treated with $200 \mu \mathrm{M}$ arsenite for $10 \mathrm{~min}$. TIA-1 immunoprecipitation yielded cellular fractions with granules whose size as measured in TEM was $150-200 \mathrm{~nm}$ (Fig. 1A,B). It should be noted that, for TEM, immunoprecipitated granule preparations were washed by three cycles of magnetic aggregation and elution; this procedure was necessary to retrieve intact granules and is less stringent than washing in $\mu$ MACS columns. Hence, both immunoprecipitated granules, labeled with one or more TIA-1-coupled beads, and a small number of other granules without beads, can be seen in Figure $1 \mathrm{~A}$.

Immunoprecipitation of FMRP (by monoclonal antibody 7G1-1, isotype IgG2a) showed that TIA-1 coimmunoprecipitates with FMRP. Mock immunoprecipitation by mouse IgG2a pulled down nearly undetectable amounts of TIA-1 and FMRP (Fig. 1C). Immunoprecipitation with magnetic beads coupled to goat IgG, or mock immunoprecipitation (no antibody, no beads) did not bring down FMRP or TIA-1. Therefore, the coimmunoprecipitation of FMRP and TIA- 1 is not attributable to nonspecific association of large FMRPcontaining protein aggregates with the SG.

After stringent washing of magneticbead coupled granules in $\mu$ MACS columns, and elution of proteins with hot SDS buffer, Western blotting established the presence of FMRP, as well as FXR1, FXR2, and NUFIP in the purified granules. We also observed the presence of TIAR, eIF4E, eIF4G, the small ribosomal subunit protein S6, poly(A)-binding protein (PABP), and HuR (Fig. 1D). These protein components are known to be present in classical stress granules (Kedersha et al., 2002). We did not observe the presence of large ribosomal proteins L3 or L28, but L4 and L7a were present. Because no FMRP or other proteins were trapped or eluted from the columns in the absence of anti-TIA-1-linked magnetic beads, the presence of L4 and L7a might suggest the presence of some of the large ribosomal proteins in stress granules under certain conditions; however, we do not consider this to be evidence for the presence of large ribosomal subunits in SG.

We measured the proportion of FMRP in stress granules after quick decapitation and rapid removal of the hippocampus. In duplicate measurements of three independent experiments with rat hippocampus, $31 \pm 0.55 \%$ (SEM) of the FMRP was found in TIA-1isolated stress granules, suggesting considerable stability of SGs.

\section{FMRP shifts between subcellular compartments in response to chemical stress}

Under stress conditions such as heat shock, FMRP shifts away from polyribosomes into untranslated stress granules (Mazroui et al., 2002). We investigated whether, in addition to heat stress, chemical stress [i.e., arsenite, which modifies protein sulfhydryl groups (Brostrom et al., 1996)] would cause FMRP to shift between polyribosomes and stress granules.

Mouse hippocampi were used for bath stimulation, with or without added arsenite. After bath stimulation of hippocampus with $200 \mu \mathrm{M}$ arsenite for $10 \mathrm{~min}$, FMRP levels in stress granules and polyribosomes were measured on Western blots. Arsenite caused a rapid redistribution of FMRP into stress granules and away from translating polyribosomes as shown in Figure 2. In control experiments, incubation for $10 \mathrm{~min}$ in DMEM without added arsenite did not significantly change the FMRP distribution.

\section{Electrode insertion}

To test the levels of physiological change caused by simple electrode insertion, we placed two concentric metal recording electrodes si- 
A

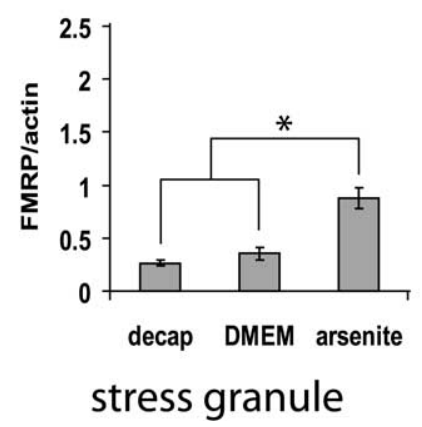

B

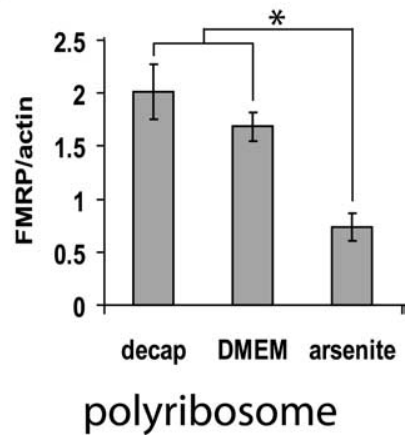

Figure 2. FMRP shifts from polyribosomes to SGs after arsenite stress. Arsenite is known to induce formation of stress granules. $A$, FMRP level in $S G s$ ( $n=4$ mice for decapitation only; $n=$ 3 mice for DMEM control; $n=5$ mice for arsenite). $\boldsymbol{B}$, FMRP level in polysomes ( $n=2$ for decapitation only; $n=3$ mice for DMEM control; $n=3$ for arsenite). "Decap only" samples are control hippocampi which were dissected out as soon as possible after decapitation and frozen on dry ice. "DMEM" indicates incubation for $10 \mathrm{~min}$ in DMEM without arsenite. Data are presented as means \pm SEM. ${ }^{*} p<0.05$.

multaneously in the PP and the DG of the hippocampus in vivo. Neuronal discharges were evoked in both PP and DG (Fig. 3A), caused by the injury from the electrode penetration. The recorded mass discharge of units in PP and DG subsided after $10 \mathrm{~s}$ (Fig. 3A). The duration of neuronal injury discharge recorded from the PP and DG ranged from 1.7 to $27.5 \mathrm{~s}$ with an average duration of $11.5 \mathrm{~s}$ [ $\pm 6.9 \mathrm{~s}(\mathrm{SD})]$ in PP and $11.2 \mathrm{~s}[ \pm 6.9 \mathrm{~s}(\mathrm{SD})]$ in $\mathrm{DG}$.

Cross-correlation analysis of injury discharges in PP and DG (within the first $6 \mathrm{~s}$ ) suggests that some DG activity was influenced by PP input mediated via a short conducting pathway and perhaps a single synapse (Fig. $3 B$ ). The average cross-correlation function estimate was $0.18 \pm 0.16(\mathrm{SD})$ and the average peak lag period (latency of correlated spikes between the PP and DG) was $4.7 \pm 1.2 \mathrm{~ms}$ (SD). Next, we examined the shifts in FMRP level in these subcellular compartments in response to the electrode insertion.

Ten minutes after simple electrode penetration and placement, FMRP was significantly elevated in the stress granules (Fig. $4 A$ ) and reduced in the polyribosomes (Fig. $4 B$ ). Because the amount of FMRP found in the cytosol was negligible, it is likely that FMRP shifted from polyribosomes into stress granules (Fig. $4 A, B)$. Note that the effect was not attributable to general anesthesia, because the levels of FMRP in the granules and polyribosomes from decapitated control rats (C1) were not significantly different from the anesthesia-only control rats (C2), and the shifts in levels of stress granule and polyribsomal FMRP were tightly time-locked to the initial electrode placement. At 20 min after electrode placement, the FMRP in stress granules began to decrease, whereas the amount of FMRP on polyribosomes increased. At $30 \mathrm{~min}, \mathrm{FMRP}$ in both stress granules and polyribosomes returned to control levels. Moreover, the contralateral (mechanically unaffected) hippocampus showed the same but not significant pattern of effect (Fig. $4 C, D$ ). Thus, there is most likely a bidirectional shuttling of FMRP between stress granules and polyribosomes; recovery was fairly rapid (30 $\mathrm{min})$ but occurred far more slowly than the electrical injury discharge.

\section{Discussion}

Stress granules are cytoplasmic microdomains formed in response to stress such as heat, UV light, or hyperosmolarity; one or more eIF2 $\alpha$ kinases [PKR (double-stranded RNA-dependent protein kinase), GCN2 (general control nonrepressed 2), HRI
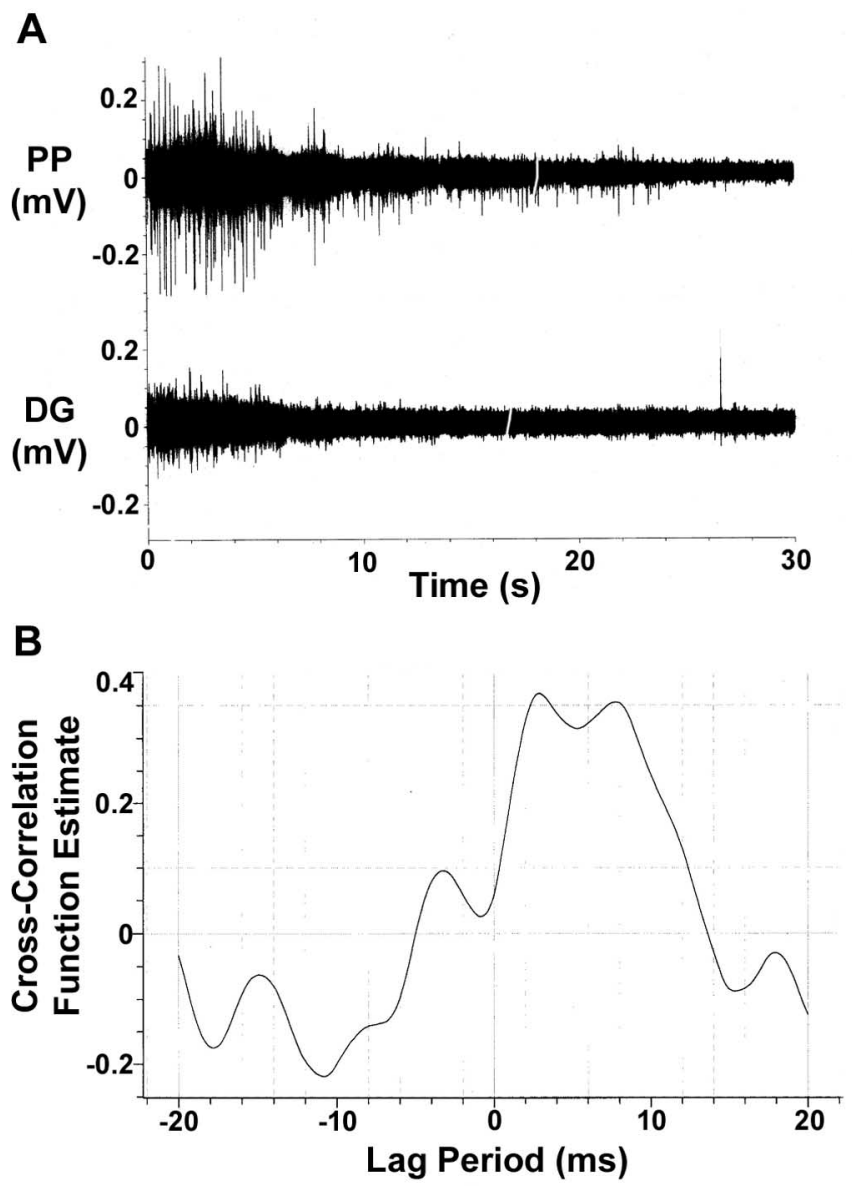

Figure 3. Recordings of multiple cell discharges in the DG and PP. $\boldsymbol{A}$, Discharges of the injured neurons were recorded from the electrodes inserted into the PP and DG of hippocampus. Mass discharges subsided after 10 s. $\boldsymbol{B}$, Cross-correlation analysis was performed to see whether the discharges recorded in the DG were influenced by the injury discharges in the PP. This example showed that (1) about one-half of the discharges in the DG are correlated to those in the PP, and (2) the lag time or latency of the spikes between the two sites is consistent with the calculated conduction time of action potentials along PP axons and the time of one synaptic delay (or latency for a presynaptic signal to cross a neurochemical synapse to initiate a postsynaptic signal).

(heme-regulated inhibitor), PERK/PEK (PKR-like endoplasmic reticulum kinase/pancreatic eIF2a kinase)] are activated, leading to phosphorylation of eIF2 $\alpha$ (Anderson and Kedersha, 2002). Phosphorylated eIF2 $\alpha$ has an increased affinity for eIF2B, a guanine nucleotide exchange factor, such that the GDP-GTP exchange is inhibited; the stores of eIF2-GTP-tRNA ${ }^{\text {met }}$ are depleted, and TIA-1 recruits preinitiation complexes (deficient in eIF2-eIF5) into stress granules. TIA-1 self-aggregation promotes the accumulation of these translationally silent complexes into stress granules, which include FMRP and additional RNAbinding proteins. They are characterized by the presence of RNAbinding proteins TIA-1, TIAR, and PABP. It is suggested that mRNPs (mRNA-proteins) are routed through these microdomains and perhaps subjected to triage (Anderson and Kedersha, 2002). Using fluorescence recovery after photobleaching, Kedersha et al. (2000) showed that both TIA-1 and PABP-1 rapidly and continuously shuttle in and out of SGs; moreover, mRNA can also shuttle between SGs and polysomes during stress. Given that $70 \%$ of green fluorescent protein (GFP)-TIA-1 can be replaced in a photobleached SG in a living cell in $\sim 4 \mathrm{~s}$, it was unexpected that antibody to TIA-1 could be successfully used for immunopurifi- 


\section{right hippocampus (electrode insertion)}

A

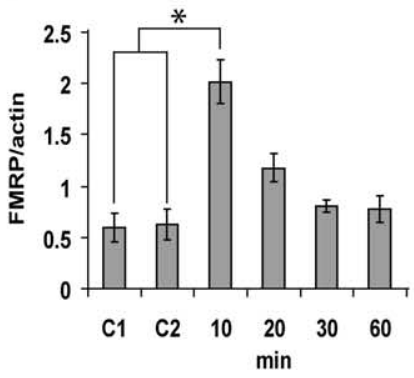

stress granule
B

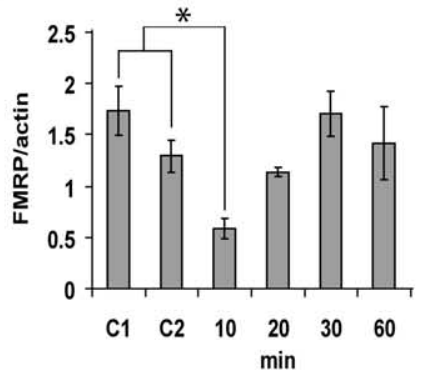

polyribosome

\section{left hippocampus (no electrode insertion)}

C

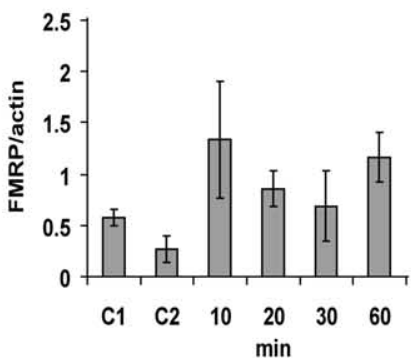

stress granule

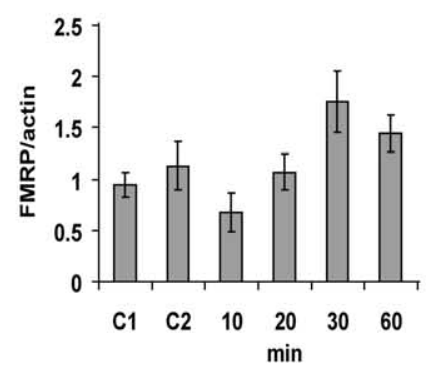

polyribosome

Figure 4. Effects of unilateral hippocampal electrode insertion: Western blot analysis of FMRP level in SGs and polyribosomes. Levels of FMRP in stress granules and polyribosomes in two control groups are shown in each panel; the hippocampus was removed for analysis after decapitation without (C1) and with (C2) anesthesia. Ten minutes after PP and DG electrodes were placed in the right hippocampus, FMRP was significantly reduced in polyribosomes $(\boldsymbol{B})$ and increased in stress granules $(\boldsymbol{A})$ in the right hippocampus. The FMRP levels in these compartments returned to the baseline after electrodes were left in the right hippocampus for $30 \mathrm{~min}$. A similar pattern of responses occurred in the contralateral hippocampus, which was not directly injured by electrode insertion and placement, but this was not statistically significant (C, FMRP level in SGs of left undamaged hippocampus; $\boldsymbol{D}$, FMRP level in polyribosomes of same). $n=4$ rats each for C $1, n=3$ for $(2, n=3$ for each duration of electrode placement. Data are presented as means \pm SEM. ${ }^{*} p<0.05$.

cation. However, in lysed cells, the conformational changes which drive SG assembly in vivo may be altered, perhaps as a result of decreased levels of chaperones such as HSP70 (heat shock protein 70). TIA-1 has a prion-related domain (PRD), and in GFP-PRD transfectants, aggregated GFP-PRD assumes a more stable conformation (Gilks et al., 2004).

This is the first quantitative description, using immunopurified SGs, of a translocation of FMRP between polyribosomes and SGs after arsenite treatment and, more importantly, of a bidirectional shift of FMRP after electrophysiological stress. We have shown that arsenite causes a shift of FMRP in addition to the shift of abortive preinitiation complexes previously described (Kedersha et al., 2002). We also examined the change of FMRP level in the polyribosome and stress granule compartments in vivo, in response to insertion of an electrode into the PP and DG of hippocampus. Ten minutes after simple electrode penetration, preexisting FMRP most likely shifted from polyribosomes into stress granules; in the course of another 10-20 min, the FMRP returned to polyribosomes. The same pattern of shifts for FMRP between stress granules and polyribosome complexes was observed in the contralateral hippocampus, suggesting that despite the absence of direct physical injury, the injury discharges transmitted through the hippocampal commissure (Voneida et al., 1981) were sufficient to trigger the cellular stress granule response. Although the injury discharge subsided after $10 \mathrm{~s}$, the shift of FMRP into stress granules lasted at least $10 \mathrm{~min}$; and resting levels were manifested only after $30 \mathrm{~min}$. Translocation of FMRP and its associated mRNAs into SGs could result in partial translational arrest during stress, whereas the movement back into polyribosomes could be a mechanism for restarting baseline translation during recovery.

There are low constitutive levels of TIA-1-containing SGs in control neurons, suggesting that they may play a role in normal regulation of protein synthesis (Kayali et al., 2005). Stressful conditions increase the assembly of larger granules (Kedersha et al., 1999). In addition to experimental electrode insertion as observed here, clinical conditions such as cerebral ischemia lead to formation of stress granules and transient inhibition of neuronal protein synthesis. As shown in a recently developed rat model for cerebral ischemia, increased formation of stress granules is re- sponsible for global protein synthesis inhibition; removal of the stress permits dissolution of stress granules by $90 \mathrm{~min}$ of reperfusion; but they persist in cells in which the induced stress leads to cell death (Kayali et al., 2005).

Costaining of FMRP and TIA-1 in cultured neurons might reveal whether they exhibit subcellular colocalization in SGs in somatic, dendritic, or spine areas. Our observations are potentially important to experimenters in setting up optimal conditions for observing physiologically relevant LTP/LTD in studies of fragile X mental retardation (for review, see Bear et al., 2004). Because of the duration and bilateral nature of the cellular injury response to electrode insertion into the hippocampus, our results imply that a 30 min waiting period should be introduced into protocols for in vivo induction of LTP or LTD.

\section{References}

Anderson P, Kedersha N (2002) Stressful initiations. J Cell Sci 115:3227-3234

Bear MF, Huber KM, Warren ST (2004) The mGluR theory of fragile X mental retardation. Trends Neurosci 27:370-377.

Brostrom CO, Prostko CR, Kaufman RJ, Brostrom MA (1996) Inhibition of translational initiation by activators of the glucose-regulated stress protein and heat shock protein stress response systems. Role of the interferon-inducible double-stranded RNA-activated eukaryotic initiation factor 2alpha kinase. J Biol Chem 271:24995-25002.

Gilks N, Kedersha N, Ayodele M, Shen L, Stoecklin G, Dember LJ, Anderson $\mathrm{P}$ (2004) Stress granule assembly is mediated by prion-like aggregation of TIA-1. Mol Biol Cell 15:5383-5398.

Huber KM, Gallagher SM, Warren ST, Bear MF (2002) Altered synaptic plasticity in a mouse model of fragile $\mathrm{X}$ mental retardation. Proc Natl Acad Sci USA 99:7746-7750.

Irwin SA, Idupulapati M, Gilbert ME, Harris JB, Chakravarti AB, Rogers EJ, Crisostomo RA, Larsen BP, Mehta A, Alcantara CJ, Patel B, Swain RA, Weiler IJ, Oostra BA, Greenough WT (2002) Dendritic spine and dendritic field characteristics of layer $\mathrm{V}$ pyramidal neurons in the visual cortex of fragile-X knockout mice. Am J Med Genet 111:140-246.

Kayali F, Montie HL, Rafols JA, DeGracia DJ (2005) Prolonged translation arrest in reperfused hippocampal cornu ammonis 1 is mediated by stress granules. Neuroscience 134:1223-1245.

Kedersha N, Cho MR, Li W, Yacono PW, Chen S, Gilks N, Golan DE, Anderson P (2000) Dynamic shuttling of TIA-1 accompanies the recruitment of mRNA to mammalian stress granules. J Cell Biol 151:1257-1268.

Kedersha N, Chen S, Gilks N, Li W, Miller IJ, Stahl J, Anderson P (2002) Evidence that ternary complex (eIF2-GTP-tRNA(i)(Met))-deficient 
preinitiation complexes are core constituents of mammalian stress granules. Mol Biol Cell 13:195-210.

Kedersha NL, Gupta M, Li W, Miller I, Anderson P (1999) RNA-binding proteins TIA-1 and TIAR link the phosphorylation of eIF-2 alpha to the assembly of mammalian stress granules. J Cell Biol 147:1431-1441.

Li J, Pelletier MR, Velazquez JP, Carlen PL (2002) Reduced cortical synaptic plasticity and GluR1 expression associated with fragile X mental retardation protein deficiency. Mol Cell Neurosci 19:138-151.

Mazroui R, Huot ME, Tremblay S, Filion C, Labelle Y, Khandjian EW (2002) Trapping of messenger RNA by Fragile X Mental Retardation protein into cytoplasmic granules induces translation repression. Hum Mol Genet 11:3007-3017.
Miyashiro KY, Beckel-Mitchener A, Purk TP, Becker KG, Barret T, Liu L, Carbonetto S, Weiler IJ, Greenough WT, Eberwine J (2003) RNA cargoes associating with FMRP reveal deficits in cellular functioning in Fmr1 null mice. Neuron 37:417-431.

Nover L, Scharf K, Neumann D (1989) Cytoplasmic heat shock granules are formed from precursor particles and are associated with a specific set of mRNAs. Mol Cell Biol 9:1298-1308.

Turner G, Webb T, Wake S, Robinson H (1996) Prevalence of fragile X syndrome. Am J Med Genet 64:196-197.

Voneida TJ, Vardaris RM, Fish SE, Reiheld CT (1981) The origin of the hippocampal commissure in the rat. Anat Rec 201:91-103.

Warren ST, Nelson DL (1994) Advances in molecular analysis of fragile X syndrome. J Am Med Assoc 271:536-542. 